\title{
Monitoring and evaluation of Integrated Disease Surveillance and Response in selected districts in Tanzania
}

\author{
S.F. RUMISHA ${ }^{1 *}$, L.E.G. MBOERA ${ }^{1}$, K.P. SENKORO ${ }^{1}$, D. GUEYE ${ }^{2}$, and P.K. MMBUJI ${ }^{3}$ \\ ${ }^{1}$ National Institute for Medical Research, P.O. Box 9653,Dar es Salaam, Tanzania \\ ${ }^{2}$ Partners for Health Reform Plus, Bethesda, Maryland 20814, USA \\ ${ }^{3}$ Ministry of Health and Social Welfare, P.O. Box 9083, Dar es Salaam, Tanzania
}

\begin{abstract}
Integrated Disease Surveillance and Response (IDSR) is a strategy developed by the World Health Organization Regional Office for Africa in 1998. The Ministry of Health, Tanzania has adopted this strategy for strengthening communicable diseases surveillance in the country. In order to improve the effectiveness of the implementation of IDSR monitoring and evaluating the performance of the surveillance system, identifying areas that require strengthening and taking action is important. This paper presents the findings of baseline data collection for the period October-December 2003 in 12 districts representing eight regions of Tanzania. The districts involved were Mbulu, Babati, Dodoma Rural, Mpwapwa, Igunga, Tabora Urban, Mwanza Urban, Muleba, Nkasi, Sumbawanga Rural, Tunduru and Masasi. Results are grouped into three key areas: surveillance reporting, use of surveillance data and management of the IDSR system. In general, reporting systems are weak, both in terms of receiving all reports from all facilities in a timely manner, and in managing those reports at the district level. Routine analysis of surveillance data is not being done at facility or district levels, and districts do not monitor the performance of their surveillance system. There was also good communication and coordination with other sectors in terms of sharing information and resources. It is important that districts' capacity on IDSR is strengthened to enable them monitor and evaluate their own performance using established indicators.
\end{abstract}

Key words: integrated disease surveillance, monitoring, evaluation, Tanzania

\section{Introduction}

Integrated Disease Surveillance and Response (IDSR) is a strategy developed by the World Health Organization Regional Office for Africa (WHO/ AFRO) in 1998. It is aimed to assist health workers to detect and respond to diseases of epidemic potential, of public health importance and those targeted for eradication and elimination. The information collected through this strategy will help district health teams to respond quickly to outbreaks, set priorities, plan interventions, and mobilize and allocate resources. The IDSR strategy links community, health facility, district, regional and national levels with the overall objective of providing epidemiological evidence for use in making decisions and implementing public health interventions for the control and prevention of communicable diseases.

Tanzania has been a leader among African countries to adopt the IDSR strategy, being the first to conduct an assessment and develop a plan of action in 1998. This was followed by the development of a work plan for integrating and strengthening disease surveillance, establishment of an IDSR Task Force (2000), preparation of the National Guidelines for Integrated Disease Surveillance and Response (2001), development of laboratory-networking guidelines (2001), and adaptation and approval of the WHO/ AFRO district analysis book (2002). The National
Guidelines for IDSR focus on 13 priority diseases, which include epidemic prone diseases (cholera, bacillary dysentery, plague, measles, yellow fever, cerebro-spinal meningitis, rabies), diseases targeted for elimination/eradication (acute flaccid paralysis, neonatal tetanus) and diseases of public health importance (diarrhoea in children $<5$ years, pneumonia in $<5$ years, malaria and typhoid) $(\mathrm{MoH}, 2001)$.

In February 2000, the National Institute for Medical Research, Tanzania spearheaded the establishment of the East African Integrated Disease Surveillance Network with the objective of creating a forum for sharing epidemiological information in the region (Rumisha et al., 2003; Mboera et al., 2004). The experienced gained by the Institute, was utilised to support the implementation of IDSR strategy in 12 selected districts in 8 regions of Tanzania.

Monitoring and evaluation (M\&E) of the project's implementation is an important component to ensure that the project is accomplishing its goals. The assessment reported in this paper focused on baseline monitoring and evaluation activities carried out before any of the districts had received training in IDSR planned to take place in April 2004. The purpose of this assessment was therefore, to gather specific information on the performance of IDSR systems in each of the districts selected. 


\section{Materials and Methods}

\section{Study area}

This work was carried out in 12 districts, which accounts for $10 \%$ of all districts in Tanzania. The districts included Babati and Mbulu (Manyara Region), Dodoma Rural and Mpwapwa (Dodoma Region), Mwanza Urban (Mwanza Region), Muleba (Kagera Region), Tabora Urban and Igunga (Tabora Region), Sumbawanga Rural and Nkasi (Rukwa Region), Tunduru (Ruvuma Region) and Masasi (Mtwara Region) .

The performance areas that were targeted for monitoring were categorised into three groups: reporting, use of surveillance data and management of the IDSR system. A total of 34 indicators were developed that cover these categories at the regional, district and health facility levels. Eight of these are based on the core indicators proposed by the World Health Organization/Regional Office for Africa (WHO/AFRO) IDSR Task Force for monitoring progress with implementation of IDSR in the African region. These are focused on the district level and are being used for self-monitoring by several countries.

\section{Study design}

At the health facility level, a sampling framework was developed that included one hospital, two health centres and $15 \%$ of dispensaries for each district. A total of 109 health facilities were visited. Within each district the selection of health facilities was made on a convenience basis with an effort to make the sample as representative as possible, taking into consideration time and transport constraints. However, in some districts the selection of health centres and dispensaries was truly random.

The main mechanisms used to collect the required data included record review and group interviews. At the district level, weekly and monthly surveillance reports submitted by all health facilities for the period October to December 2003, report tracking tools, results of data analysis, minutes of meetings conducted, schedules and reports for health education and other activities, and Comprehensive Council Health Plans were reviewed and documented. At the facility level, register, copies of weekly and monthly reports at facility for October to December 2003, results of data analysis, schedules and reports for community outreach activities and standard case definitions were reviewed. At the regional level, a review was done on weekly and monthly reports submitted by all districts in the region for the period October to December 2003.
Group interviews were organized to gather information about activities related to IDSR that had occurred during the period under review. The group format was used because the purpose was not to evaluate individual performance, but rather to assess IDSR activities as a whole. Participants were often asked to provide examples to support their responses. This served as a means of verifying that the question had been understood and attempting to assure the validity of the responses provided, rather than just relying on yes/no answers. At the district level, the interview involved key members of the council (district) health management teams (CHMT), including the District Medical Officer (DMO), the District Health Officer (DHO), who in some of the districts was the IDSR Focal Person, the Health Management Information System (HMIS) Focal Person, the Expanded Programme on Immunisation (EPI) Focal Person, and others involved in IDSR. In areas were the IDSR Focal Person was someone other than the DHO, then this person was also included in the interview. At health facilities the in-charge and one other staff person, when available, were interviewed. At the regional level, key members of the Regional Health Management Team including the Regional Medical Officer (RMO), Regional Health Officer (RHO), IDSR focal person, HMIS focal person and EPI focal person were involved in the group interviews.

Data collection was carried out from JanuaryMarch 2004.Data collectors provided feedback to each health facility on the results of the M\&E. At the end of data collection in a district a debriefing meeting was also held with members of the CHMT to discuss facility and district results.

\section{Data analysis}

Data entry and check files were prepared in Epi Info version 6 for the interview and report accuracy data collection instruments. Data from the record reviews was entered into Excel spreadsheets. The data were then transferred to Excel and STATA version 7 to make one master file containing all information from all forms. Frequency distributions were calculated for all variables. The master database was then cleaned and analysed using conventional statistical methods.

\section{Results}

\section{Completeness and timeliness of surveillance reporting}

A total of 109 health facilities were included in the monitoring and evaluation of the IDSR strategy. Of 
these, 12 were hospitals, 24 health centres and 74 dispensaries. Except for Sumbawanga Rural District, hospitals were surveyed in all the districts.

Dodoma Rural and Masasi districts were not using the weekly surveillance reports during this period and were thus not included in weekly calculations. Different deadlines were found in the districts for monthly and weekly reports. The weekly reporting period was not standard in most of the districts, although this was less of a problem for monthly reports. Even within the same district the facilities were found to have different days of starting and ending the report week (for example, from Tuesday to Monday or Friday to Thursday). Local deadlines for each district were used to assess the timeliness of the reports from health facilities. For the October December period under review, three monthly reports and 13 weekly reports were expected.

Total reporting completeness for all districts was 33\% (range: $7-71 \%$ ) for monthly reports and 19\% (range: 1-48\%) for weekly reports (Figure 1). In general, completeness was higher for monthly reports than for weekly reports. None of the districts met the target of receiving $80 \%$ of expected reports for the quarter. Tunduru had the highest rate of monthly reporting, but one of the lowest weekly rates. Nkasi was the most consistent, with approximately $50 \%$ of its facilities reporting on a weekly and monthly basis (Figure 1). When inquiring about poor reporting performance in some facilities, it was found that the periods of poorest performance often correlated to the periods when the person responsible for reporting was on leave.

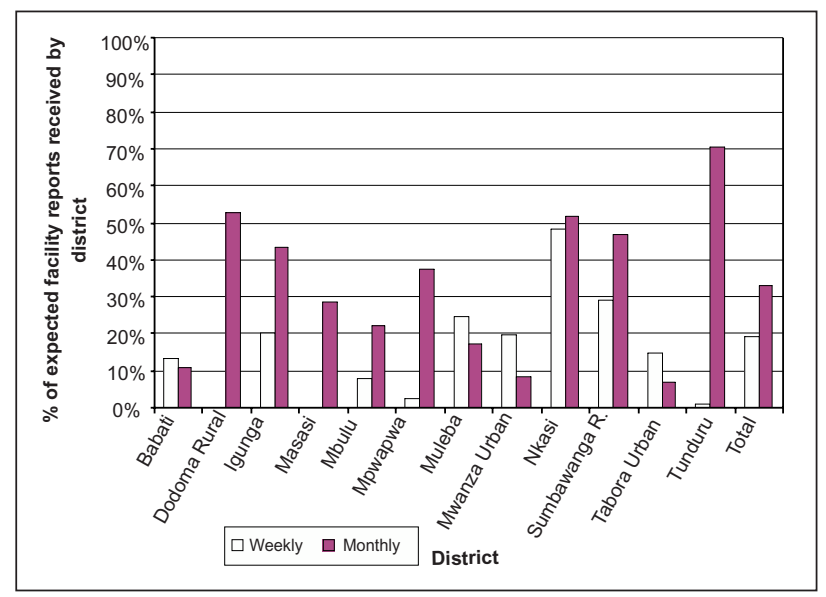

Figure 1: Completeness of health facility reporting to district
Completeness for district reports to the region was much higher, 66\% (range: $31-90 \%$ ) for weekly reports and $80 \%$ (range: $50-100 \%$ ) for monthly reports overall (Figure 2). It should be noted that these results cover all districts within the regions, not only those participating in IDSR activities. Kagera, Rukwa and Tabora regions all achieved $100 \%$ coverage of monthly reporting.

Eleven of the twelve districts were tracking timeliness, either directly on the reports or on a tracking form. No data were available for Dodoma Rural and Masasi districts because they were not using weekly reports, while Tunduru was not tracking timeliness for weekly reports. Some districts recorded the actual dates that reports were received, while others only marked timely or late, which did not allow for independent verification.

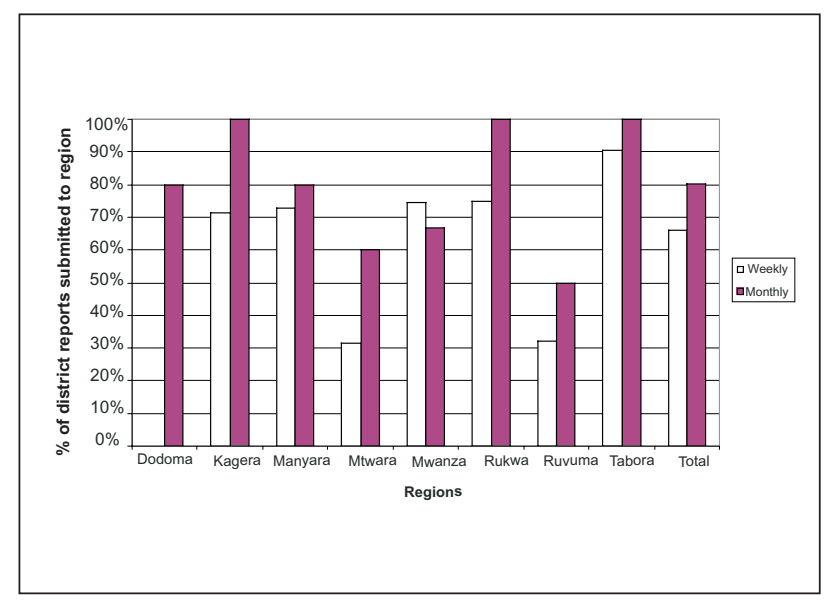

Figure 2: Completeness of district reporting to region

The overall timeliness of reporting was only $8 \%$ (range 0-19\%) for weekly reports and 24\% (range: 3 $56 \%$ ) for monthly reports (Figure 3 ). Timeliness was measured as the percentage of expected reports received by the due date. Tunduru had the highest rate of timeliness for monthly reports (56\%), while Muleba and Mwanza Urban had the highest rates for weekly reports, at $18 \%$ and $19 \%$, respectively. Igunga had $0 \%$ timeliness for weekly reports. None of the districts reached the target of $80 \%$ of reports received on time. 


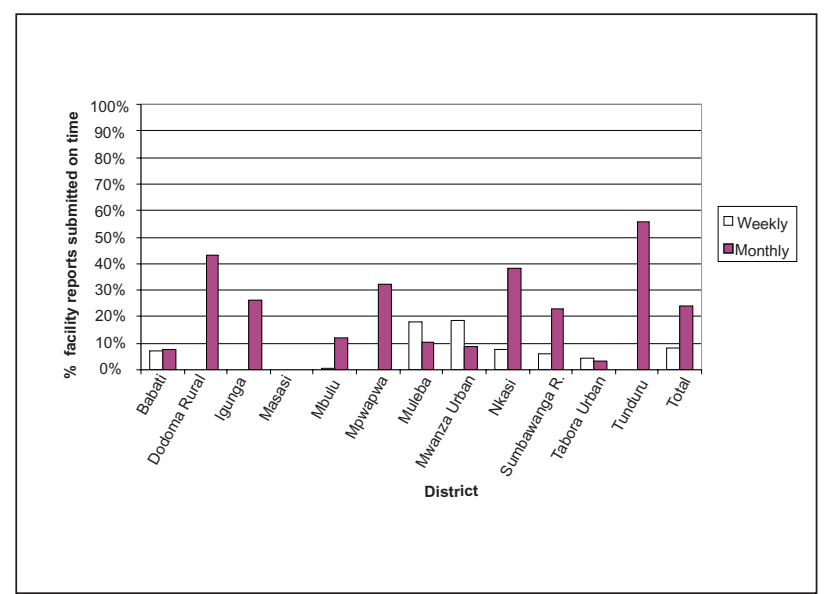

Figure 3: Timeliness of health facility reporting to district

Examining the performance of all facilities combined over time it was observed that the coverage and timeliness followed similar patterns during the quarter. The highest point for each was reached during the week of October 22, with significant declines during the holiday period at the end of the quarter.

District reporting to the region was more efficient than facility reporting to the district. The deadlines for the district reports to the region were mentioned to be Thursday for weekly reports and the $15^{\text {th }}$ of the coming month for the monthly reports. On average $47 \%$ (range: $1-74 \%$ ) of expected weekly reports were on time, while the it was $60 \%$ (range: $0-94 \%$ ) for monthly reports. Half of the regions met or exceeded the target of $80 \%$ timeliness for monthly reports. Our findings showed that weekly reporting lags behind in most regions, with the exception of Mwanza Urban (Figure 4). The results for Mtwara region were so low due primarily to the fact that several reports from the districts were not available at the regional office; among the reports reviewed only one was found to be timely.

In general, field visits for data collection revealed that data management and organization were weak at the district and health facility levels. Most districts had reports organized into weekly and monthly folders by year, but these were not always maintained. None were organized by facility, which could facilitate monitoring of reporting performance. In some cases it took most of the day for all of the required reports to be obtained because they were filed in many different places. Different formats were being used for weekly and monthly reports and many reports were hand-drawn on notebook paper. Some facilities were using the old Essential Drug Programme and
Infectious Disease Week Ending forms for sending reports to the district level. Lack of standardized reporting formats resulted in inconsistency of reporting between districts.

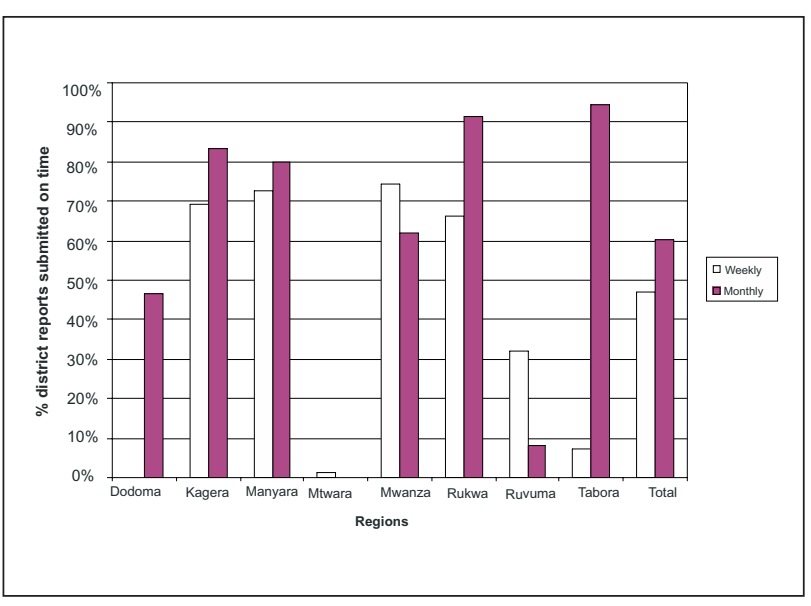

Figure 4: Timeliness of district reporting to region

In several instances weekly and monthly reports were found at the health facilities but not at the district level, suggesting problems in the reporting chain. Several possible explanations exist: (i) the reports were not actually sent from the facility, (ii) the reports were sent with local transport or by other means but never delivered to the district, (iii) the reports were delivered to the district office but not to the right person (particularly possible when the district office and district hospital were co-located), and (iv) the reports were delivered to the appropriate person at the district but then lost. There was also difficulty in obtaining copies of reports for health facilities that use radio calls to transmit their reports to the district. Many facility staff noted that they had a heavy reporting burden complicated by inconsistent requirements (HMIS, IDSR, other programmes). For example, the HMIS reports combine pneumonia, bronchitis, and other respiratory ailments under Acute Respiratory Infections, while the IDSR report asks for only pneumonia and severe pneumonia. Different reporting systems use different age categories and some require reporting by sex. The result is that facility staff must have a very good system for reviewing registers and tallying their data, or must do it multiple times to meet the different criteria for different programmes.

\section{Accuracy of reports}

Standard case definitions were not always used for recording diagnoses in registers. In many instances, plasmodiasis was recorded instead of malaria; bloody 
diarrhoea was recorded instead of bacillary dysentery. In addition, a number of uncommon and/or unknown abbreviations were found in registers, leading to incorrect tallying and reporting of data. Problems were observed wherein patients appeared to be registered more than once. For example, a patient was registered in the out-patient department book and also registered in the in-patient department book. Most of the District hospitals were not submitting their weekly and monthly reports to the district. There were several cases of reportable diseases found in registers that had not been included in monthly health facility reports. There was no standard inpatient register available so each facility improvised a hand-drawn register using a blank record book. Although HMIS Book 1 had guidance on what to include in the inpatient register, there was no standardization among facilities, or even from one page to the next. In addition, registers were often incomplete, with information such as date, age, sex, diagnosis, treatment or outcome missing. Some health facilities in the districts were found not to be using the HMIS system at all; a few did not know what the books look like and what is supposed to be done. Thus data were not recorded in a standard way and incorrect reports were submitted. This was mainly found in hospitals and in private health facilities.

Many of the discrepancies were due to the fact that facility reports had been received after the district had already submitted its report to the region; thus the tally of cases from reports found at the time of data collection was often higher than the number reported to the region. This highlights the importance of having good coverage and timeliness of reporting in order to have an accurate picture of the disease situation. Given that health facilities were not always using the same deadlines for submitting reports, it was difficult for districts to compile meaningful reports to send to the regions. Due to this, information received was sometimes found to be included in a different reporting period, sometimes combined with data for the coming week or month.

\section{Use of surveillance data}

Analysis of data at the district level was quite weak. Five of the twelve districts reported doing any type of trend analysis for IDSR priority diseases on a monthly or quarterly basis. Only two districts (Mbulu and Nkasi) stated that they did some type of trend analysis for malaria. WHO/AFRO recommends two specific types of analysis - tracking of monthly malaria inpatient cases and deaths, and long-term trend analysis (i.e. year to year) of malaria, both for children aged less than five years - and that these analyses include data from the previous three months. Only Nkasi district had both types of analysis, but they were not current. In fact, Nkasi had trend analysis for all of the weekly reported diseases. All but two of the districts (Sumbawanga and Mwanza Urban) had lists of the top ten diseases in their catchment area. Mbulu district had displayed person analysis for priority diseases and three districts (Dodoma Rural, Masasi and Mpwapwa) had maps with disease cases marked.

At the facility level, 32\% of facilities reported doing any type of trend analysis for priority diseases. However, only $4 \%$ had graphs of monthly malaria cases and deaths, representing one facility each in Dodoma, Igunga, Muleba and Sumbawanga districts, and none had long-term trends. The graphs in three of the four facilities contained current data. Listing of top ten diseases was less common at the facility level, available only at $7 \%$ of the facilities (representing Dodoma, Igunga, Masasi and Mwanza Urban districts). One facility each in Igunga and Muleba districts had maps with disease cases marked.

Health personnel at both the district and regional levels were asked about their knowledge of IDSR indicators, whether they had reviewed those indicators during the previous three months, and whether they had taken any actions as a result of the review. Knowledge of the indicators found in the National Guidelines for IDSR was quite weak at both levels. These indicators include: timeliness and coverage of reporting, case fatality rates, proportion of outbreaks notified to the district within 24 hours, proportion of outbreaks with laboratory confirmation. Seven of the eight regions reported being familiar with the indicators, but only two could provide specific examples to support this (Dodoma and Ruvuma). At the district level, three districts (Mpwapwa, Dodoma and Muleba) reported knowing the indicators but only one district (Dodoma) provided examples.

Five regions reported that their RHMTs met in the previous quarter to review indicators that tell about the performance of the IDSR system, but only Mtwara and Ruvuma regions provided evidence to support this. Both of these regions also took actions based on their indicator review, including sending letters and guidelines to the districts on how to prepare reports, notifying districts of poor performance, suggesting ways to resolve communications problems, and assuring availability of reporting forms at all districts. None of the districts met to review their IDSR indicators. 
District teams were asked whether they had used IDSR data to provide justification for their plans and actions, as well as to monitor the impact of their actions. All of the districts except one (Muleba) stated that they had used data for planning purposes. IDSR data used as the basis for (i) introducing fare reimbursement for health workers to bring reports to the district in order to increase coverage (Babati district); (ii) information about animal bites was used to order drugs from the Medical Stores Department (Tunduru); (iii) data on increased diarrhoea cases in two wards was used to develop a programme for toilet construction (Masasi); (iv) an increased number of malaria cases led to the distribution and promotion of insecticide-treated nets (ITNs) (Tabora). All but one of the districts that had used data for planning also stated that they had used data for monitoring their activities, either in the previous quarter (4 districts) or the past year. Igunga had used data for planning but not monitoring.

\section{Planning and implementation of IDSR activities}

A key measure of the institutionalization and sustainability of IDSR is whether related activities and their associated costs are included in district plans and budgets. If an activity is not documented in the district plan and sufficient funding allocated, it is not likely to occur. As part of the district interview, participants were asked about whether their district had planned for any of the following activities: (i) supervision visits to health facilities, (ii) meetings to review or report on IDSR activities, (iii) IDSR training, and (iv) prevention activities of priority infectious diseases. Council Comprehensive Health Plan (CCHP) and other documented sources (such as supervision schedules or activity calendars) were reviewed to verify the information. Districts were also asked about their implementation of planned activities. Three of the districts (Dodoma, Igunga and Mpwapwa) were able to meet the criteria for planning and implementing all of the suggested activities, including implementation of $80 \%$ or more of planned supervision visits. All other districts met three or four of the criteria.

All of the districts had included supervision visits to their health facilities in their CCHPs. For the
October - December 2003 period, half of the districts planned to carry out supervision visits at a rate of one visit per facility per month. Another two districts planned to visit each facility once per quarter. The remaining two districts planned to visit only half of their facilities during the quarter. On average, districts implemented $33 \%$ of their planned supervision visits for the previous quarter. The best performing districts were Dodoma, Igunga and Tabora. However, the first two of these districts also had the fewest visits planned, proportional to the number of facilities. Among the districts that planned monthly visits, Babati and Mbulu were able to implement the most, (about 60\%). Most of the districts that planned quarterly supervision visits had generally high implementation rates. In four of the districts documentation on how many supervision visits had been carried out was poor or not available.

Six of the districts had planned meetings that reported on or reviewed IDSR activities. All of these meetings were held, and three other districts also held meetings that had not been included in their CCHPs. It was observed, during discussion that most of the CHMT members had a timetable of monthly CHMT meeting, while some districts held quarterly meetings specifically to discuss IDSR and Mwanza held public meetings on the control of waterborne diseases. Babati, Muleba and Tabora districts did not hold any meeting in the previous quarter at which IDSR activities were discussed.

Ten of the districts had planned training related to IDSR for the previous year and carried out their plans. Examples of training topics included data collection for infectious disease and reporting, use of line lists and measles outbreak reporting (Mwanza), disease surveillance and data management (Igunga), infectious disease prevention (Nkasi) and malaria case management (Tabora).

All of the districts had included activities to prevent priority infectious diseases in their 2003 CCHP, and all but one district (Masasi) carried out prevention activities during the previous quarter. These included promotion of insecticide-treated nets, vaccination campaigns and environmental sanitation activities. 
Table1: The percentage of facility with availability of tools and job aids by district

\begin{tabular}{|c|c|c|c|c|c|c|c|c|c|c|c|c|c|}
\hline & 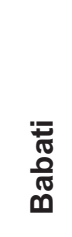 & $\begin{array}{l}\stackrel{\mathbb{Z}}{\varepsilon} \\
\stackrel{0}{0} \\
\stackrel{0}{\circ}\end{array}$ & 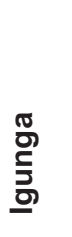 & $\begin{array}{l}\bar{n} \\
\mathbb{D} \\
\mathscr{D} \\
\mathbb{D} \\
\Sigma\end{array}$ & $\begin{array}{l}\text { 즐 } \\
\text { 을 }\end{array}$ & $\begin{array}{l}\frac{\pi}{3} \\
\frac{2}{10} \\
\frac{10}{2} \\
\sum\end{array}$ & $\frac{\frac{\pi}{0}}{\frac{0}{0}}$ & $\begin{array}{l}\mathbb{N} \\
\text { N } \\
\text { N } \\
\stackrel{3}{3} \\
\Sigma\end{array}$ & $\begin{array}{l}\bar{y} \\
\stackrel{\varpi}{Z} \\
\bar{z}\end{array}$ & 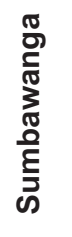 & 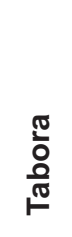 & 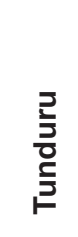 & 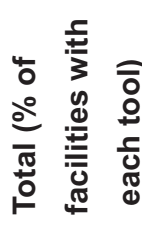 \\
\hline \multicolumn{14}{|l|}{ Criteria } \\
\hline Register & 100 & 100 & 100 & 90 & 100 & 100 & 100 & 100 & 100 & 100 & 100 & 100 & 99 \\
\hline $\mathrm{CIF} \longrightarrow \mathrm{AFP}$ & 38 & 50 & 14 & 10 & 14 & 22 & 33 & 8 & 0 & 0 & 0 & 13 & 17 \\
\hline $\mathrm{CIF}-\mathrm{NNT}$ & 50 & 67 & 0 & 10 & 14 & 22 & 33 & 8 & 29 & 8 & 0 & 50 & 24 \\
\hline CIF - Measles & 25 & 50 & 14 & 10 & 14 & 22 & 33 & 8 & 0 & 0 & 0 & 38 & 18 \\
\hline CIF — Generic & 25 & 25 & 0 & 10 & 0 & 0 & 0 & 0 & 0 & 0 & 0 & 13 & 7 \\
\hline Weekly forms & 100 & 67 & 43 & 30 & 71 & 89 & 83 & 23 & 100 & 92 & 75 & 38 & 65 \\
\hline Monthly forms & 75 & 92 & 57 & 80 & 71 & 100 & 67 & 38 & 86 & 100 & 50 & 88 & 76 \\
\hline \multicolumn{14}{|l|}{ Standard case } \\
\hline definitions & 75 & 83 & 100 & 40 & 71 & 89 & 83 & 77 & 71 & 92 & 88 & 88 & 79 \\
\hline \multicolumn{14}{|c|}{ Proportion of expected tools available for each district } \\
\hline & 61 & 67 & 41 & 35 & 45 & 56 & 54 & 33 & 48 & 49 & 39 & 53 & 48 \\
\hline
\end{tabular}

Key: CIF = Case investigation form; AFP $=$ acute flaccid paralysis; NNT = Neonatal Tenanus

\section{Availability of tools/job aids for IDSR}

The essential tools and job aids include the facility register, case investigation forms (disease-specific and generic), weekly and monthly reporting forms, and standard case definitions. Facility staffs were asked if they had each of these items, and were asked to show them to the data collectors in order to receive credit. Overall, less than $2 \%$ of health facilities had all of the tools available, while $22 \%$ had more than half of the 8 tools. On average, facilities had four of the tools available. These were most likely to be the register, weekly and monthly reporting forms, and standard case definitions (Table 1). All of the different case investigation forms were consistently in short supply. Only 7\% of the facilities had the generic case investigation form, which covers several diseases. There was no significant variability among the districts in terms of the expected proportion of tools that were actually available, with a low of $35 \%$ in Masasi and a high of $67 \%$ in Dodoma Rural. However, there was a significant difference between the availability of the forms per district $(P<0.0001)$. There was notable variability among facilities within some districts.

\section{Feedback}

This exercise examined feedback on reports at three levels - from the Ministry of Health to the region, from the region to the district, and from the district to the health facility - always from the receiver's point of view. Four criteria were used to evaluate feedback during the previous quarter: receipt of technical information or updates on policies that related to infectious disease reporting, investigation or response (such as a technical bulletin on cholera specimen collection procedures or new reporting deadlines); receipt of reports showing data for districts/facilities combined or comparing districts/facilities; receipt of information about the quality of weekly and monthly surveillance reports submitted; and receipt of assistance in carrying out IDSR activities (such as instruction on properly completing a form during a supervision visit).

The regions were asked about whether they had received feedback from the Ministry of Health on IDSR issues. Among the eight regions, only three received feedback on new policy and guidelines, one region received aggregated data, four received feedback on quality of IDSR reports and two received assistance on how to conduct IDSR tasks. Manyara Region did not report receiving any type of feedback from the Ministry of Health, while Mtwara, Mwanza and Tabora regions received two types of feedback.

Districts received feedback on different criteria during the October- December period. While five of the districts reported receiving more than two types of feedback, only one district (Nkasi) received all four. Nkasi and Sumbawanga are in the same region (Rukwa), but Sumbawanga did not report receiving any updates or assistance with IDSR tasks. Districts 
in Kagera, Manyara and Mwanza regions did not receive any of the specified feedback during the period. About half of the districts responded that they had received feedback regarding the quality of their surveillance reports and assistance in carrying out their IDSR responsibilities. Feedback of aggregated or comparative data was reported by only four districts. Some examples of feedback received included: Policies/technical updates; aggregated/comparative data; quality of reports; and assistance with IDSR responsibilities.

Feedback from districts to health facilities was significantly weaker than feedback from regions to districts. Overall, $40 \%$ of health facilities interviewed reported that they did not receive any type of feedback (as defined above) during the preceding quarter; this varied from a low of $8 \%$ in Dodoma Rural to a high of $92 \%$ in Mwanza. No facilities received all four types of feedback, while one-third of health facilities received one type of feedback. Approximately 30\% of facilities received either technical/policy updates or assistance from the district, while only $6.5 \%$ received aggregated or compiled data. Mpwapwa performed the best, providing at least two type of feedback to $22 \%$ of its facilities.

Feedback to different facilities within a district was often variable. For example, the Babati District Hospital reported having received three types of feedback, while all but one of the other seven facilities interviewed reported zero. The case is similar for Masasi, Mbulu, and Igunga districts. Nkasi had designed a specific form for providing feedback to all health facilities on a quarterly basis. This consisted of a list of all health facilities and months (January to December). The forms were found at health facilities during the data collectors' visits.

\section{Discussion}

IDSR has been adopted as a national strategy in Tanzania since 1998, but it has not been effectively rolled out in most of the country. As the results and observations of this study show, many of the key tasks in the strategy are not being carried out as planned, particularly at the lower levels of the health system. Data quality is important, beginning at the level where it is generated. If data is not valid and reliable at this level, neither is anything at the higher levels. Weekly reporting is weak, with some districts not using this system at all. Monthly reporting performance is better, perhaps due to the overlap with periods of salary collection. Similar findings have been reported by other workers in Tanzania (Nsubuga et al., 2002;
Mboera et al., 2001a,b; Mboera et al., 2004; Mghamba et al., 2004).

Completeness and timeliness are key indicators of reporting performance. These are defined as the proportion of expected reports received (completeness), and the proportion of expected reports received on time (timeliness). In this study, reports were considered late if they had not been received by the established deadline. Reports received after the deadlines and those for which timeliness could not be assessed were grouped together. Only when a district has received reports from all facilities on the expected date can it be confident about knowing the true disease situation and make decisions accordingly. Poor timeliness in reporting from health facilities to the respective district has been reported in a previous study by Nsubuga et al. (2002). Poor timeliness reporting in Tanzania has been attributed to lack of adequate information exchange facilities and systems that facilitate rapid access to information available at one level or both levels of health delivery system. For instance, reporting between facility and district levels is constrained by the lack of effective and efficient means of communication (Kajeguka \& Mboera, 2003; Mboera, 2004; Mboera et al., 2005). There is need to emphasize the use of information communication technology to improve surveillance reporting in the country. Moreover, health workers should be involved in a participatory manner to improve their timely reporting improvising available means of communication as shown in a recent study in Dodoma, Tanzania (Mboera et al., 2005).

In several instances weekly and monthly reports were found at the health facilities but not at the district level, suggesting problems in the reporting chain. Several possible explanations exist: (i) the reports were not actually sent from the facility; (ii) the reports were sent with local transport or by other means but never delivered to the district; (iii) the reports were delivered to the district office but not to the right person (particularly possible when the district office and district hospital were co-located), and; (iv) the reports were delivered to the appropriate person at the district but then lost.

Lack of uniformity in recording at health facilities in the districts under study is likely to be due to lack of standard case definitions. A similar situation has been reported in recent years by other workers in Tanzania. For instance, in assessing the structure and performance for five infectious disease surveillance systems in Tanzania (Nsubuga et al., 2002) found out that standardized case definitions were used for only 3 of 21 infectious diseases. In another study, it has 
been reported that health workers rely mostly on their clinical skills rather than applying standard case definition leading to inconsistent recording diagnoses (Franco et al., 2003; Mghamba et al. (2004). Standard case definition job aids are important tools with regard to proper detection of cases. Improper detection of cases gives insufficient and unreliable tracking of diseases.

With regard to data analysis and interpretation, it needs to be done at all levels of disease surveillance right from community to national and international levels. The kind of analysis will vary according to requirements and what can be done at that particular level. At whatever level, data are normally analysed by time, place and person. Time analysis enables the comparison of cases reported for the current time (week/month/year) with the number received in the previous time (week/month/year). This enables the detection of any abrupt or long-term changes in disease occurrence. Low utilisation of surveillance data has been associated with understaffing, lack of skills and low motivation by health workers (Mwangu \& Otito, 2000). It has been emphasised that epidemiological data analysis and interpretation need to be done at all levels (community, facility, district, national) of disease surveillance (Mghamba et al., 2004).

Feedback and supervision have also been found to be poor in this study. In a previous study, only $42 \%$ of the health facility in Tanzania received supervision or feedback (Nsubuga et al., 2002). Feedback has been singled out as one of the most important tool for developing health workers and improving their performance. A data management tool (District Analysis Book) has been incorporated in the current IDSR system (Mghamba et al., 2004).

In general, data management and organization were weak at the district and facility levels. Data management, and particularly file organization, is a problem at both facility and district levels. This is a barrier to seeing the true picture of reporting performance. Related to this is the mechanism for submitting reports, particularly the issue of reports submitted by radio-call not being individually documented. Lack of standardization of reporting forms makes it difficult to compile data. It is important that the districts have a specific person for receiving and sending of reports, set specific time for receiving and proper documentation at district and facility level. Sentinel centres for collection of reports from remote health facilities can be made to facilitate reporting as already being practised in Dodoma (Mboera et al., 2005). The understaffing, lack of skills by some health workers and motivation was found to explain the low utilization of data (Mwangu \& Otito, 2000). Nsubuga et al (2002) also found that although data analysis was carried out in all five surveillance systems, incidence and prevalence were rarely calculated and moreover trends in disease outbreaks were also seldom tracked. This has an implication with regard to planning and taking action using evidence based information and in this case the use of analysed data.

There is a problem of centralization of responsibilities at some health facilities. This is particularly evident when the in-charge is away and the remaining staffs are not aware of responsibilities related to IDSR (such as completing appropriate forms/reports at the appropriate time). Multiple and diverse reporting requirements pose a problem for health workers. An integration of these programmes and systems to reduce the burden of tasks assigned, which are sometimes repeated will likely to solve some of these problems.

District and facility personnel were very interested in receiving immediate feedback about their performance, and numerous problem-solving discussions were held. The use of case investigation forms was better for vaccine-preventable diseases, likely due to the strong Expanded Program on Immunization (EPI) in most districts. The unit of Reproductive and Child Health use these forms under EPI, which are Measles, Polio and Neonatal tetanus. This is done and coordinated by programme and there is a specific focal person that is why most of the filled forms found were of these diseases.

Data analysis at both district and facility levels is weak. At health facility, completion of HMIS book 2 was found to be a great step on the process of analysis. In a study in Iringa Urban District it was observed that $44.5 \%$ of the health workers were not utilising the information (including surveillance) collected through HMIS (Kagonji, 2005). This means that there's close relationship between practice of health workers and information utilization, and hence practice of health workers affects information utilization. Creation of culture of using information and motivation of health worker are very important aspect to strengthening this activity.

In conclusion, it is important that the capacities of health workers at all levels to implement IDSR are strengthened if we are to have a sustainable implementation of disease surveillance in Tanzania. Unfortunately, after 7 years since the adoption of the IDSR strategy by Tanzania, there is limited quantitative evidence that suggests that the IDSR intervention has led to improvements in perceived 
availability of quality data, capacity to perform data analysis, motivation to perform data analysis, motivation to use analyzed data, and perceived value of analyzed data (Eisele et al., 2006). The existing barriers (including overburdened health facility staff; poor communication; poor laboratory capacity; poor incentives; poor organizational capacity; and insufficient financial resources) to an effective IDSR need to be immediately addressed.

\section{Acknowledgements}

The authors want to particularly thank all the members of the Council and Regional Health Management Teams in the regions and districts of Babati and Mbulu (Manyara), Dodoma Rural and Mpwapwa (Dodoma), Igunga and Tabora Urban (Tabora), Nkasi and Sumbawanga Rural (Rukwa), Masasi (Mtwara), Tunduru (Ruvuma), Mwanza Urban (Mwanza) and Muleba (Kagera) for their support. The authors are grateful to Mtumwa Bakari, Akili Kalinga, Levina Lema, Benjamin Mayala, Jonathan Mcharo, Godwin Nkya, Dominick Shauri and Joseph Swila for their excellent field assistance. Rogers Rindeni and Fagason Mduma are thanked for their assistance in data entry. This work received financial assistance from USAIDTanzania and USAID-Global Bureau/Office of Health, Infectious Diseases and Nutrition.

\section{References}

Eisele, T., Hotchkiss, D., Bingham, A., Silvestre, E., Gueye, D., Banke, K., Mmbuji, P. \& Mboera, L.E.G. (2006) Assessing the Effectiveness of an Intervention Package for Improving Analysis and Response of the Infectious Disease Surveillance System in Tanzania. Bethesda, MD: The Partners for Health Reformsplus Project, Abt Associates Inc.

Franco, L.M., Fields, R., Mmbuji, P.K.L., Posner, S., Mboera, L.E.G., Jimmerson, A., Senkoro, K.P., Rumisha, S.F., Shayo, E.H. \& Mwami, J.A. (2003) Situation Analysis of Infectious Disease Surveillance in two Districts in Tanzania, 2002. Bethesda, MD: The Partners for Health Reformsplus Project, Abt Associates Inc.

Kajeguka, A.C. \& Mboera, L.E.G. (2003) Information and communication technology: options for strengthening integrated disease surveillance and response at district level in Tanzania. Tanzania Health Research Bulletin 5, 61-67.

Mboera, L.E.G. (2004) Disease surveillance in Tanzania: an integrated approach in the management and control of communicable diseases. Proceedings of the $19^{\text {th }}$ Annual Joint Scientific Conference of the National Institute for Medical Research, Arusha International Conference Centre, Arusha, Tanzania, March 1517, 2004.

Mboera, L.E.G., Rumisha, S.F. \& Kitua, A.Y. (2001a) Strategic approach for strengthening national and regional disease surveillance system: The East African example. Tanzania Health Research Bulletin 3 (2), 6-9.

Mboera, L.E.G., Rumisha, S.F., Magesa, S.M. \& Kitua, A.Y. (2001b) Utilisation of Health Management Information System in disease surveillance in Tanzania. Tanzania Health Research Bulletin 3 (2), 15-18.

Mboera, L.E.G., Rumisha, S.F., Mwanemile, E.J., Mziwanda, E. \& Mmbuji, P. (2005) Enhancing disease surveillance reporting using public transport in Dodoma District, central Tanzania. Tanzania Health Research Bulletin 7, 201-205.

Mboera, L.E.G., Rumisha, S.F., Senkoro, K.P., Kamugisha, M.L. \& Kitua, A.Y. (2004) East African Integrated Disease Surveillance Network 2001-2004. Technical Report submitted to the Rockefeller Foundation, November 2004. National Institute for Medical Research, Dar es Salaam, Tanzania

Mghamba, J.M., Mboera, L.E.G., Krekamoo, W., Senkoro, K.P., Rumisha, S.F., Shayo, E.H. \& Mmbuji, P. (2004) Challenges of implementing Integrated Disease Surveillance and Response strategy using the current Health Management Information System in Tanzania. Tanzania Health Research Bulletin 6, 57-63.

Mwangu, M.A. \& Otito, C.K. (2000). HMIS implementation at the district level in the context of the Health Sector Reforms: Challenges and future prospects. Proceedings of the $18^{\text {th }}$ Annual Scientific Conference of the Tanzania Public Health Association, November 22-25, 1999, Dodoma, Tanzania. Pp 111-119. 
Nsubuga, P., Eseko, N., Wuhib, T., Ndayimirije, N., Chungong, S. \& McNabb, S. (2002) Structure and performance of infectious disease surveillance and response, United Republic of Tanzania, 1998. Bulletin of the World Health Organization 80, 196-202.
Rumisha, S.F., Mboera, L.E.G. Kamugisha, M.L., Kalumuna, A., Amri, M. \& Kitua, A.Y. (2003) Pattern and distribution of communicable diseases in border districts of Bukoba and Tanga, Tanzania. Tanzania Health Research Bulletin 5, 19-23. 